\title{
Phase and TV Based Convex Sets for Blind Deconvolution of Microscopic Images
}

\author{
Mohammad Tofighi, Onur Yorulmaz, Kivanç Köse, Deniz Cansen Yıldırım, Rengül Çetin-Atalay, and A. Enis Çetin, \\ Fellow, IEEE
}

\begin{abstract}
In this paper, two closed and convex sets for blind deconvolution problem are proposed. Most blurring functions in microscopy are symmetric with respect to the origin. Therefore, they do not modify the phase of the Fourier transform (FT) of the original image. As a result blurred image and the original image have the same FT phase. Therefore, the set of images with a prescribed FT phase can be used as a constraint set in blind deconvolution problems. Another convex set that can be used during the image reconstruction process is the Epigraph Set of Total Variation (ESTV) function. This set does not need a prescribed upper bound on the Total Variation (TV) of the image. The upper bound is automatically adjusted according to the current image of the restoration process. Both the TV of the image and the blurring filter are regularized using the ESTV set. Both the phase information set and the ESTV are closed and convex sets. Therefore they can be used as a part of any blind deconvolution algorithm. Simulation examples are presented.
\end{abstract}

Index Terms-Blind deconvolution, epigraph sets, inverse problems, projection onto convex sets.

\section{INTRODUCTION}

A wide range of deconvolution algorithms has been developed to remove blur in microscopic images in recent years [1]-[17]. In this article, two new convex sets are introduced for blind deconvolution algorithms. Both sets can be incorporated to any iterative deconvolution and/or blind deconvolution method.

One of the sets is based on the phase of the Fourier transform (FT) of the observed image. Most point spread functions

Manuscript received March 15, 2015; revised August 05, 2015, October 01, 2015, and October 26, 2015; accepted October 27, 2015. Date of publication November 20, 2015; date of current version January 21, 2016. This work was supported by the Turkish Scientific and Technical Research Council (TUBITAK), under Project 113E069. M. Tofighi and O. Yorulmaz contributed equally and the names are listed in alphabetical order. The guest editor coordinating the review of this manuscript and approving it for publication was Prof. Michael Unser.

M. Tofighi, O. Yorulmaz, and A. E. Çetin are with the Department of Electrical and Electronics Engineering, Bilkent University, 06800 Ankara, Turkey (e-mail: tofighi@ee.bilkent.edu.tr; yorulmaz@ee.bilkent.edu.tr; cetin@bilkent. edu.tr).

K. Köse is with the Dermatology Service, Memorial Sloan Kettering Cancer Center, New York, NY 10022 USA (e-mail: kosek@mskcc.org).

D. C. Yildırım is with the Department of Molecular Biology and Genetics, Bilkent University, 06800 Ankara, Turkey (e-mail: deniz.yildirim@bilkent.edu. tr).

R. Çetin-Atalay is with the Bioinformatics Department, Graduate School of Informatics, Middle East Technical University, 06800 Ankara, Turkey (e-mail: rengul@metu.edu.tr).

Color versions of one or more of the figures in this paper are available online at http://ieeexplore.ieee.org.

Digital Object Identifier 10.1109/JSTSP.2015.2502541
(PSF) in x-y plane of microscopes are symmetric with respect to origin. Therefore, Fourier transform of such functions do not have any phase. As a result, FT phase of the original image and the blurred image have the same phase. The set of images with a prescribed phase is a closed and convex set and projection onto this convex set is easy to perform in Fourier domain.

The second set is the Epigraph Set of Total Variation (ESTV) function. Total variation (TV) value of an image can be limited by an upper-bound to stabilize the restoration process. In fact, such sets were used by many researchers in inverse problems [13], [18]-[23]. In this paper, the epigraph set of the TV function will be used to automatically estimate an upper-bound on the TV value of a given image. This set is also a closed and convex set. Projection onto ESTV function can be also implemented effectively. ESTV can be incorporated into any iterative blind deconvolution algorithm.

Another contribution of this article is that the ESTV set is applied onto the blurring functions during iterative deconvolution algorithms. Blurring functions are smooth functions, therefore their total variation value should not be high.

Image reconstruction from Fourier transform phase information was first considered in 1980's [24]-[27] and total variation based image denoising was introduced in 1990's [28]. However, FT phase information and ESTV have not been used in blind deconvolution problem to the best of our knowledge.

Recently, Fourier phase information is used in image quality assessment and blind deblurring by Leclaire and Moisan [29], in which phase information is used to define an image sharpness index, and this index is used as a part of a deblurring algorithm. In this article FT phase is directly used during the blind deconvolution of fluorescence (FL) microscopic images.

The paper is organized as follows. In Section II, we review image reconstruction problem from FT phase and describe the convex set based on phase information. In Sections III, we describe the Epigraph set of the TV function. We modify AyersDainty blind deconvolution method by performing orthogonal projections onto FT phase and ESTV sets in Section IV. We present our experimental results in Section V and conclude the article in Section VI.

\section{Convex Set Based on the Phase of FOURIER TRANSFORM}

In this section, we introduce our notation and describe how the phase of Fourier transform can be used in deconvolution problems. 


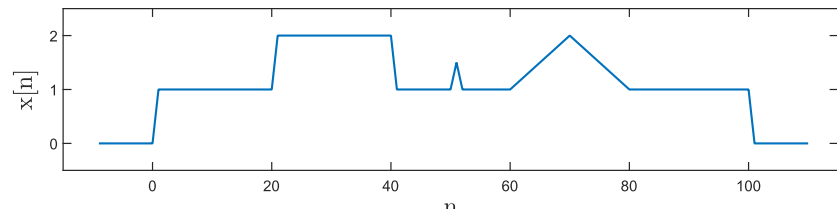

(a)

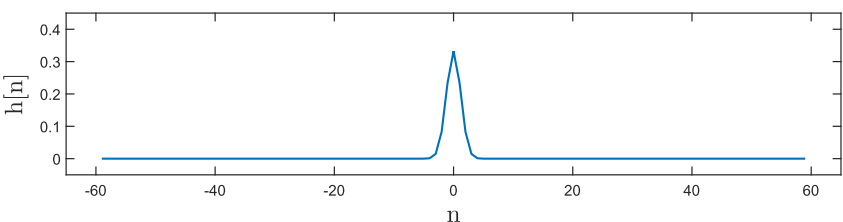

(b)

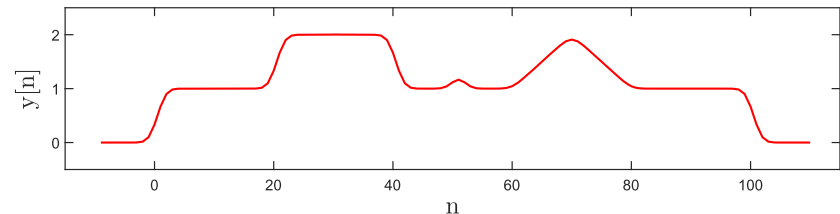

(c)

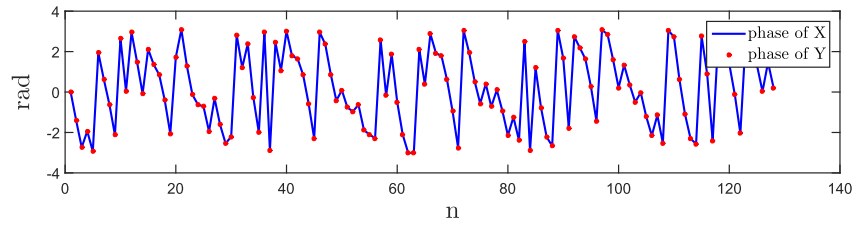

(d)

Fig. 1. (a) A 1-D signal $x$, (b) Gaussian filter $h$ with $\sigma=1.2$, (c) filtered signal $y=h * x$ and, (d) FT phase of $x$ and $y$ obtained using an FFT with size 128 .

Let $x_{o}\left[n_{1}, n_{2}\right]$ be the original image and $h\left[n_{1}, n_{2}\right]$ be the blurring function representing a slice of the 3-D point spread function. The observed image $y$ is obtained by the convolution of $h$ with $x_{0}$ :

$$
y\left[n_{1}, n_{2}\right]=h\left[n_{1}, n_{2}\right] * x_{o}\left[n_{1}, n_{2}\right],
$$

where $*$ represents the two-dimensional convolution operation. The discrete-time Fourier transform $Y$ of $y$ is, therefore, given by

$$
Y\left(w_{1}, w_{2}\right)=H\left(w_{1}, w_{2}\right) X_{o}\left(w_{1}, w_{2}\right) .
$$

When $h\left[n_{1}, n_{2}\right]$ is symmetric with respect to origin $\left(h\left[n_{1}, n_{2}\right]=(0,0)\right) H\left(w_{1}, w_{2}\right)$ is real. Our zero phase assumption is $H\left(w_{1}, w_{2}\right)=\left|H\left(w_{1}, w_{2}\right)\right|$. Blurring functions satisfying this assumption includes Gaussian blurs and uniform discs. Therefore, phase of $Y\left(w_{1}, w_{2}\right)=\left|Y\left(w_{1}, w_{2}\right)\right| e^{\left(j \measuredangle Y\left(w_{1}, w_{2}\right)\right)}$ and $X_{o}\left(w_{1}, w_{2}\right)=\left|X_{0}\left(w_{1}, w_{2}\right)\right| e^{\left(j \measuredangle X_{o}\left(w_{1}, w_{2}\right)\right)}$ are the same:

$$
\measuredangle Y\left(w_{1}, w_{2}\right)=\measuredangle X_{o}\left(w_{1}, w_{2}\right),
$$

for all $\left(w_{1}, w_{2}\right)$ values.

In Fig. 1, a one-dimensional (1-D) example is shown. The original signal $x$ is shown in Fig. 1(a). The signal $y$ is obtained by convolving $x$ with $h$, which is a Gaussian filter. FT Phase plots of $x$ and $y$ are the same as shown in Fig. 1(d).

Based on the above observation the following set can be defined:

$$
\mathcal{C}_{\phi}=\left\{x\left[n_{1}, n_{2}\right] \mid \measuredangle X\left(w_{1}, w_{2}\right)=\measuredangle X_{o}\left(w_{1}, w_{2}\right)\right\},
$$

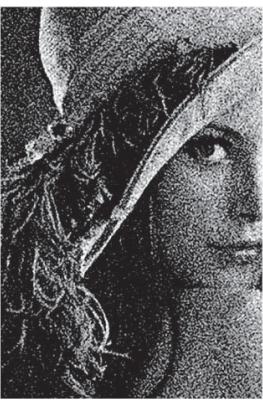

(a)

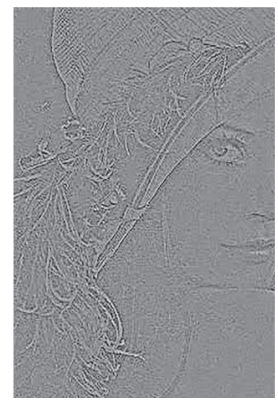

(b)

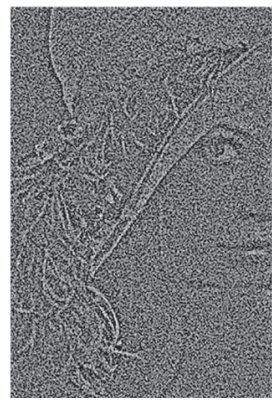

(c)
Fig. 2. (a) noisy "Lena" image, (b) Phase only version of the "Lena" image, and (c) phase only version of the noisy "Lena" image.

which is the set of images whose FT phase is equal to a given prescribed phase $\measuredangle X_{o}\left(w_{1}, w_{2}\right)$. It can easily be shown that this set is closed and convex in $\mathbb{R}^{N_{1}} \times \mathbb{R}^{N_{2}}$, for images of size $N_{1} \times N_{2}$.

Projection of an arbitrary image $x$ onto $\mathcal{C}_{\phi}$ is implemented in Fourier domain. Let the FT of $x$ be $X\left(w_{1}, w_{2}\right)=$ $\left|X\left(w_{1}, w_{2}\right)\right| e^{j \phi\left(w_{1}, w_{2}\right)}$. The FT $X_{p}$ of its projection $x_{p}$ is obtained as follows:

$$
X_{p}\left(w_{1}, w_{2}\right)=\left|X\left(w_{1}, w_{2}\right)\right| e^{j \measuredangle X_{o}\left(w_{1}, w_{2}\right)},
$$

where the magnitude of $X_{p}\left(w_{1}, w_{2}\right)$ is the same as the magnitude of $X\left(w_{1}, w_{2}\right)$ but its phase is replaced by the prescribed phase function $\measuredangle X_{o}\left(w_{1}, w_{2}\right)$. After this step, $x_{p}\left[n_{1}, n_{2}\right]$ is obtained using the inverse FT. The above operation is implemented using the FFT and implementation details are described in Section IV.

Obviously, projection of $y$ onto the $\operatorname{set} \mathcal{C}_{\phi}$ is the same as itself. Therefore, the iterative blind deconvolution algorithm should not start with the observed image. Image reconstruction from phase (IRP) has been extensively studied by Oppenheim and his coworkers [24]-[27]. IRP problem is a robust inverse problem. In Fig. 2, phase only version of the well-known Lena image is shown. The phase only image is obtained as follows:

$$
v=\mathcal{F}^{-1}\left[C e^{j \phi\left(w_{1}, w_{2}\right)}\right]
$$

where $\mathcal{F}^{-1}[\cdot]$ represents the inverse Fourier transform, $C$ is a constant and $\phi\left(w_{1}, w_{2}\right)$ is the phase of Lena image. Edges of the original image are clearly observable in the phase only image. Therefore, the set $\mathcal{C}_{\phi}$ contains the crucial edge information of the original image $x_{o}$.

When the support of $x_{o}$ is known, it is possible to reconstruct the original image from its phase within a scale factor. Oppenheim and coworkers developed Papoulis-Gerchberg type iterative algorithms from a given phase information. In [26] support and phase information are imposed on iterates in space and Fourier domains in a successive manner to reconstruct an image from its phase.

In blind deconvolution problem the support regions of $x_{o}$ and $y$ are different from each other. Exact support of the original image is not precisely known; therefore, $\mathcal{C}_{\phi}$ is not sufficient by itself to solve the blind deconvolution problem. However, it can be used as a part of any iterative blind deconvolution method.

When there is observation noise, (1) becomes:

$$
\mathbf{y}_{o}=\mathbf{y}+\nu,
$$


where $\boldsymbol{\nu}$ represents the additive noise. We use bold face letters and underlined bold face letters for $N$ dimensional vectors and $N+1$ dimensional vectors, respectively. In this case, phase of the observed image is obviously different from the phase of the original image. Luckily, phase information is robust to noise as shown in Fig. 2(c) which is obtained from a noisy version of Lena image. In spite of noise, edges of Lena are clearly visible in the phase only image. Gaussian noise with variance $\sigma=30$ is added to Lena image in Fig. 2(a). Fig. 2(b) is obtained from the original Lena image and Fig. 2(c) is obtained from the phase of noisy Lena image, respectively.

FTs of some symmetric blurring functions may take negative values for some $\left(w_{1}, w_{2}\right)$ values. In such $\left(w_{1}, w_{2}\right)$ values, phase of the observed image $Y\left(w_{1}, w_{2}\right)$ differs from $X\left(w_{1}, w_{2}\right)$ by $\pi$. Therefore, phase of $Y\left(w_{1}, w_{2}\right)$ should be corrected as in phase unwrapping algorithms. Or some of the $\left(w_{1}, w_{2}\right)$ values around $\left(w_{1}, w_{2}\right)=(0,0)$ can be used during the image reconstruction process. It is possible to estimate the main lobe of the FT of the blurring function from the observed image. Phase of FT coefficients within the main lobe are not effected by a shift of $\pi$.

In this article, the set $\mathcal{C}_{\phi}$ will be used as a part of the iterative blind deconvolution schemes developed by Dainty et al. [30] and Fish et al. [31], together with the epigraph set of total variation function which will be introduced in the next section.

\section{EPIGRAPh Set of Total Variation Function}

Bounded total variation is widely used in various image denoising and related applications [18], [23], [32]-[35]. The set $\mathcal{C}_{\text {ESTV }}$ of images whose TV values is bounded by a prescribed number $\epsilon$ is defined as follows:

$$
\mathcal{C}_{\mathrm{ESTV}}=\{\mathbf{w}: \mathrm{TV}(\mathbf{w}) \leq \epsilon\},
$$

where TV of an image is defined, in this paper, as follows:

$$
\begin{aligned}
\mathrm{TV}(\mathbf{w})=\sum_{i, j=1}^{N_{1}} \mid \mathrm{w}[i+1, j] & -\mathrm{w}[i, j] \mid \\
& +\sum_{i, j=1}^{N_{2}}|\mathrm{w}[i, j+1]-\mathrm{w}[i, j]| .
\end{aligned}
$$

This set is a closed and convex set in $\mathbb{R}^{N_{1} \times N_{2}}$. Set $\mathcal{C}_{\text {ESTV }}$ can be used in blind deconvolution problems. But the upper bound $\epsilon$ has to be determined somehow a priori.

In this article we increase the dimension of the space by one and consider the problem in $\mathbb{R}^{N_{1} \times N_{2}+1}$. We define the epigraph set of the TV function:

$$
\mathcal{C}_{\mathrm{ESTV}}=\left\{\underline{\mathbf{w}}=\left[\mathrm{w}^{T}, z\right]^{T} \mid \mathrm{TV}(\mathbf{w}) \leq z\right\},
$$

where $T$ is the transpose operation.

The concept of the epigraph set is graphically illustrated in Fig. 3. Since $T V(\mathbf{w})$ is a convex function in $\mathbb{R}^{N_{1} \times N_{2}}$, then the set $\mathcal{C}_{\mathrm{ESTV}}$ is closed and convex in $\mathbb{R}^{N_{1} \times N_{2}+1}$. In (10) one does not need to specify a prescribed upper bound on TV of an image. An orthogonal projection onto the set $\mathcal{C}_{\text {ESTV }}$ reduces the total variation value of the image, as graphically illustrated in Fig. 3, because of the convex nature of the TV function. Let $\mathbf{v}$ be an $N=N_{1} \times N_{2}$ dimensional image to be projected onto the set

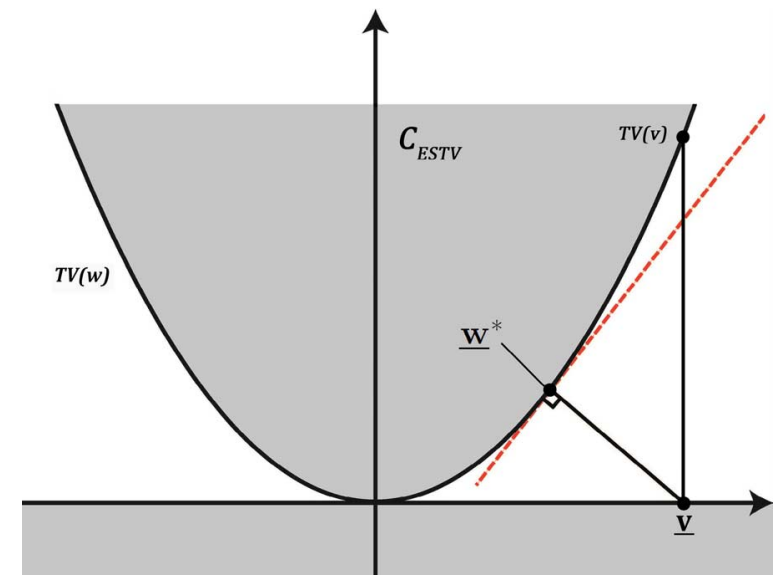

Fig. 3. Graphical representation of the orthogonal Projection onto the Epigraph Set of TV function (PES-TV), defined in (11). The observation vector $\underline{\mathbf{v}}=\left[\mathbf{v}^{T}, 0\right]^{T}$ is projected onto the set $\mathcal{C}_{\mathrm{ESTV}}$, which is the epigraph set of TV function.

$\mathcal{C}_{\text {ESTV }}$. In orthogonal projection operation, we select the nearest vector $\underline{\mathbf{w}}^{\star}$ on the set $\mathcal{C}_{\mathrm{ESTV}}$ to $\underline{\mathbf{v}}$. The projection vector $\mathbf{w}^{\star}$ of an image $\mathbf{v}$ is defined as:

$$
\underline{\mathbf{w}}^{\star}=\arg \min _{\underline{\mathbf{w}} \in \mathcal{C}_{\mathrm{ESTV}}}\|\underline{\mathbf{v}}-\underline{\mathbf{w}}\|^{2},
$$

where $\mathbf{v}=\left[\mathbf{v}^{T}, 0\right]$. The projection operation described in (11) is equivalent to:

$$
\underline{\mathbf{w}}^{\star}=\left[\begin{array}{c}
\mathbf{w}^{\star} \\
\mathrm{TV}\left(\mathbf{w}^{\star}\right)
\end{array}\right]=\arg \min _{\underline{\mathbf{w}} \in \mathcal{C}_{\mathrm{ESTV}}}\left\|\left[\begin{array}{l}
\mathbf{v} \\
0
\end{array}\right]-\left[\begin{array}{c}
\mathbf{w} \\
\mathrm{TV}(\mathbf{w})
\end{array}\right]\right\|^{2},
$$

where $\underline{\mathbf{w}}^{\star}=\left[\mathbf{w}^{\star T}, \mathrm{TV}\left(\mathbf{w}^{\star}\right)\right]$ is the projection of $\left[\mathbf{v}^{T}, 0\right]$ onto the epigraph set. The projection $\underline{w}^{\star}$ must be on the boundary of the epigraph set. Therefore, the projection must be on the form $\left[\mathbf{w}^{\star T}, \mathrm{TV}\left(\mathbf{w}^{\star}\right)\right]$. Equation (12) becomes:

$$
\underline{\mathbf{w}}^{\star}=\left[\begin{array}{c}
\mathbf{w}^{\star} \\
\mathrm{TV}\left(\mathbf{w}^{\star}\right)
\end{array}\right]=\arg \min _{\underline{\mathbf{w}} \in \mathcal{C}_{\mathrm{ESTV}}}\|\mathbf{v}-\mathbf{w}\|^{2}+\mathrm{TV}(\mathbf{w})^{2} .
$$

It is also possible to use $\lambda \mathrm{TV}($.$) as a the convex cost function$ and (13) becomes:

$$
\underline{\mathbf{w}}^{\star}=\left[\begin{array}{c}
\mathbf{w}^{\star} \\
\mathrm{TV}\left(\mathbf{w}^{\star}\right)
\end{array}\right]=\arg \min _{\underline{\mathbf{w}} \in \mathcal{C}_{\mathrm{ESTV}}}\|\mathbf{v}-\mathbf{w}\|^{2}+\lambda^{2} \mathrm{TV}(\mathbf{w})^{2} .
$$

The solution of (11) can be obtained using the method that we discussed in [23], [36]. The solution is obtained in an iterative manner and the key step in each iteration is an orthogonal projection onto a supporting hyperplane of the set $\mathcal{C}_{\text {ESTV }}$.

In current TV based denoising methods [33] the following cost function is used:

$$
\mathbf{w}^{*}=\arg \min _{\underline{\mathbf{w}} \in \mathcal{C}_{\mathrm{ESTV}}}\|\mathbf{v}-\mathbf{w}\|^{2}+\lambda \mathrm{TV}(\mathbf{w}) .
$$

However, we were not able to prove that (15) corresponds to a non-expansive map or not. On the other hand, minimization problem in (13) and (14) are the results of projection onto convex sets, as a result they correspond to non-expansive maps [5], [18], [32], [37]-[42]. Therefore, they can be incorporated into any iterative deblurring algorithm without affecting the convergence of the algorithm. 


\section{How to InCORporate $\mathcal{C}_{\text {ESTV }}$ AND $\mathcal{C}_{\phi}$ Into A DEBLURRING METHOD}

In this section, we present our implementation to integrate phase and TV based convex sets approach into Blind AyersDainty Method with Phase and ESTV sets.

One of the earliest blind deconvolution methods is the iterative space-Fourier domain method developed by Ayers and Dainty [30]. In this approach, iterations start with a $x_{o}[n]=$ $x_{o}\left[n_{1}, n_{2}\right]$, where we introduce a new notation to specify equations $[n]=\left[n_{1}, n_{2}\right]$. For example, we rewrite (1) as follows:

$$
y[n]=h[n] * x_{o}[n] .
$$

The method successively updates $h[n]$ and $x[n]$ in a Wiener filter-like equation. Here is the $i$ th step of the algorithm:

1) Compute $\widehat{X}_{i}(w)=\mathcal{F}\left\{\widehat{x}_{i}[n]\right\}$, where $\mathcal{F}$ represents the FT operation and $w=\left(w_{1}, w_{2}\right)$, with some abuse of notation.

2) Estimate the blurring filter response using the following equation

$$
\widetilde{H}_{i}(w)=\frac{Y(w) \widehat{X}_{i}^{*}(w)}{\left|\widehat{X}_{i}(w)\right|^{2}+\alpha /\left|\widehat{H}_{i}(w)\right|^{2}},
$$

where $\alpha$ is a small real number.

3) Compute $\widetilde{h}_{i}[n]=\mathcal{F}^{-1}\left\{\widetilde{H}_{i}(w)\right\}$.

4) Impose the positivity constraint and finite support constraints on $\widetilde{h}_{i}[n]$. Let the output of this step be $\widehat{h}_{i}[n]$.

5) Compute $\widehat{H}_{i}(w)=\mathcal{F}\left\{\widehat{h}_{i}[n]\right\}$.

6) Update the image

$$
\tilde{X}_{i}(w)=\frac{Y(w) \widehat{H}_{i}^{*}(w)}{\left|\widehat{H}_{i}(w)\right|^{2}+\alpha /\left|\widehat{X}_{i}(w)\right|^{2}} .
$$

7) Compute $\widetilde{x}_{i}[n]=\mathcal{F}^{-1}\left\{\widetilde{X}_{i}(w)\right\}$.

8) Impose spatial domain positivity and finite support constraint on $\widetilde{x}_{i}[n]$ to produce the next iterate $\widehat{x}_{i+1}[n]$.

Iterations are stopped when there is no significant change between successive iterates. We can easily modify this algorithm using the convex sets defined in Section II and III.

In the proposed algorithm, the phase information is imposed on the current iterate as follows:

$$
\widehat{\widehat{X}}_{i}(w)=\left|\widehat{X}_{i}(w)\right| e^{j \measuredangle Y(w)},
$$

where $\measuredangle Y(w)$ is the phase of $Y(w)$. This step is the projection onto the set $\mathcal{C}_{\phi}$. We also introduce a new step to Ayers and Dainty's algorithm as follows: project $\overline{\bar{x}}_{i}[n]$ onto the set $\mathcal{C}_{\text {ESTV }}$ to obtain $\widehat{x}_{i+1}[n]$ as follows:

$$
\widehat{x}_{i+1}[n]=\arg \min _{x_{i} \in \mathcal{C}_{\mathrm{ESTV}}}\left\|\overline{\bar{x}}_{i}[n]-x_{i}[n]\right\|^{2} .
$$

Similarly the updated blur filter $\widehat{\widehat{h}}_{i}[n]$ is obtained by projecting $\widehat{h}_{i}[n]$ onto the set $\mathcal{C}_{\mathrm{ESTV}}$ as follows:

$$
\widehat{\widehat{h}}[n]=\arg \min _{h \in \mathcal{C}_{\text {ESTV }}}\|\widehat{h}[n]-h[n]\|^{2} .
$$

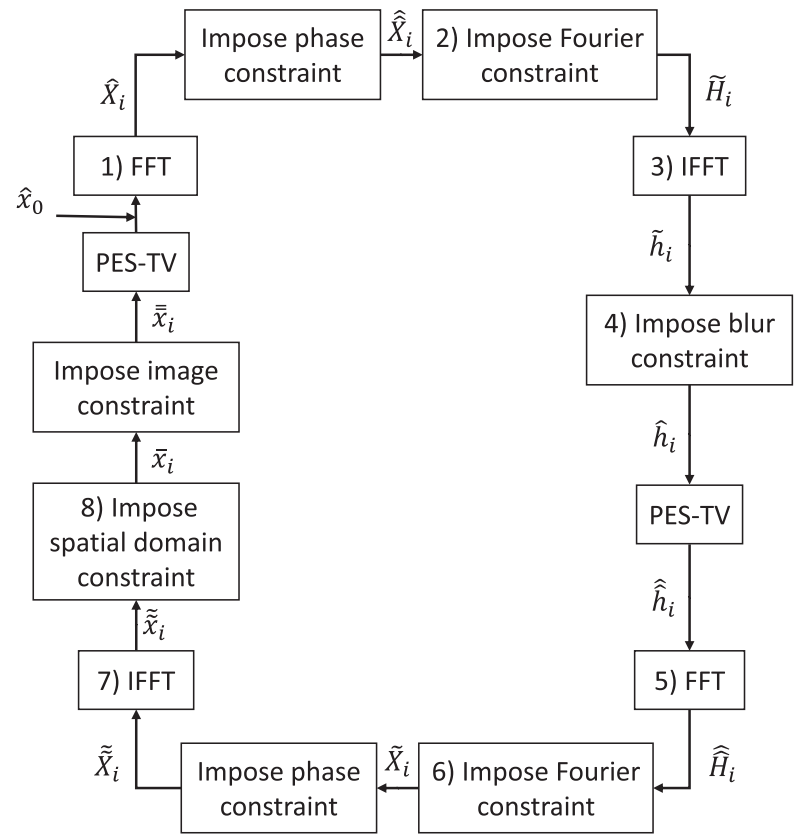

Fig. 4. Flowchart of the proposed algorithm based on Ayers-Dainty method PES-TV stands for Projection onto the Epigraph Set of TV function.

The steps 1-8) will also change according to the proposed algorithm. The flowchart of the proposed algorithm is shown in Fig. 4.

Since the filter is a zero-phase filter in microscopic image analysis $h\left[n_{1}, n_{2}\right]=h\left[-n_{1},-n_{2}\right]=h\left[-n_{1}, n_{2}\right]=h\left[n_{1},-n_{2}\right]$ this condition is also imposed on the current iterate in Step 4.

The term "support" refers to the extent of the image. Let us assume that the 2D image is $N_{1} \times N_{2}$. Its phase can be computed by $L_{1} \times L_{2}$ DFT, where $L_{1} \geq N_{1}$ and $L_{2} \geq N_{2}$. Inverse FFT may produce nonzero values outside the $N_{1} \times N_{2}$ region. In this case we make the values of pixels outside the support region $\left(N_{1} \times N_{2}\right.$ region) simply zero.

Global convergence of Ayers-Dainty method has not been proved. In fact, we experimentally observed that it may diverge in some FL microscopy images. Projections onto convex sets are non-expansive maps [42]-[44], therefore, they do not cause any divergence problems in an iterative image debluring algorithm.

\section{EXPERIMENTAL RESULTS}

We first start with the example given in "http://www. optinav.com/Iterative-Deconvolution.htm" [45], which is linked by the EPFL 3D Deconvolution in microscopy web-page "http://bigwww.epfl.ch/deconvolution/?p=bio". In Fig. 5, the original image is shown in part (a). The blurred image with a Gaussian with $\sigma=6$ is shown in Fig. 5(b). This image has a PSNR $=24.05 \mathrm{~dB}$. The image deblurred using the non-blind method in [45] is shown in Fig. 5(c), PSNR $=22.43 \mathrm{~dB}$. We downloaded the restored image from [45]. The relatively low PSNR value may be due to a shift of pixels. The restoration result of ordinary Ayers-Dainty algorithm is shown in Fig. 5(d) with a PSNR $=24.86 \mathrm{~dB}$. The restoration result of the Ayers-Dainty method with phase information has a higher PSNR $=24.91 \mathrm{~dB}$ (Fig. 5(e)). The white cable or stripe on the ground is barely visible. This cable is not visible in Figs. 5(b)-(d). Result of the Ayers-Dainty method and ESTV 


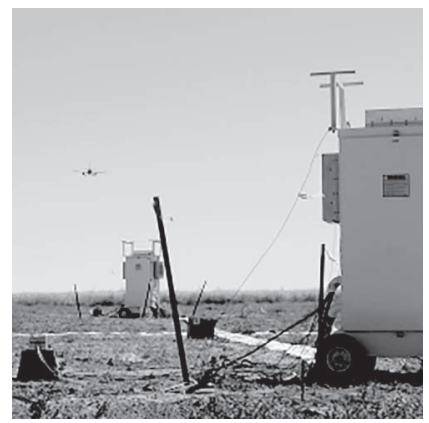

(a)

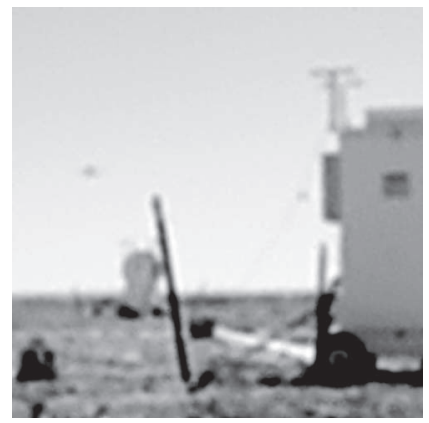

(c)

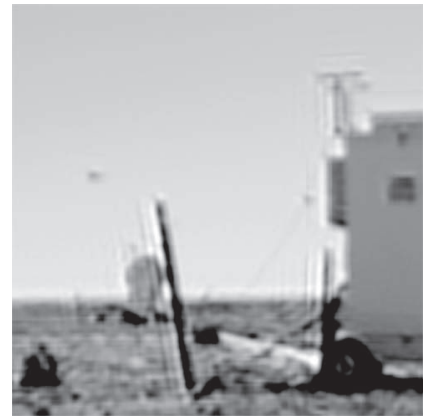

(e)

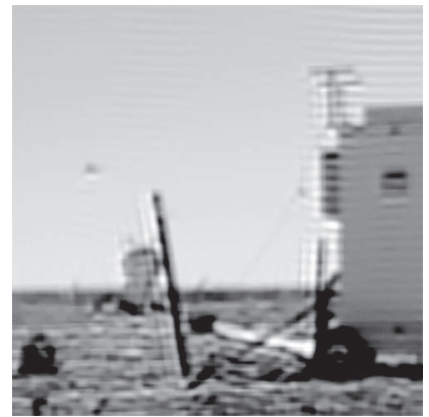

(g)

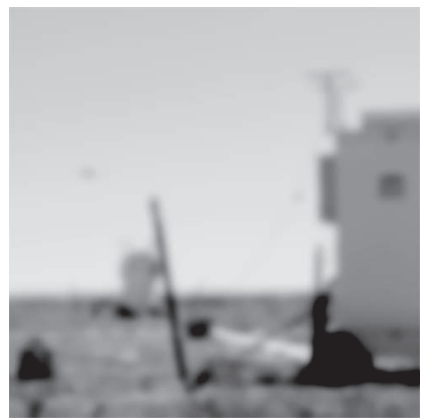

(b)

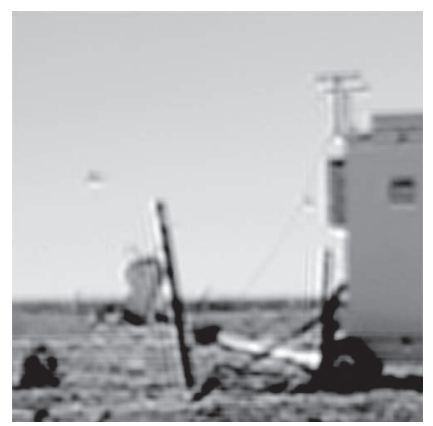

(d)

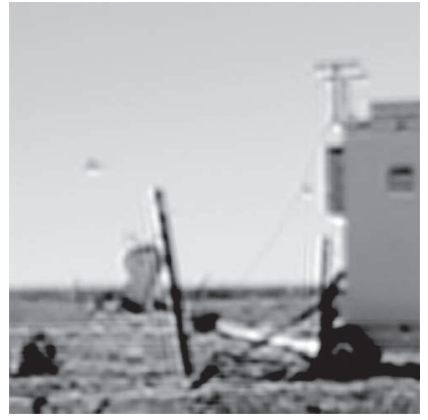

(f)

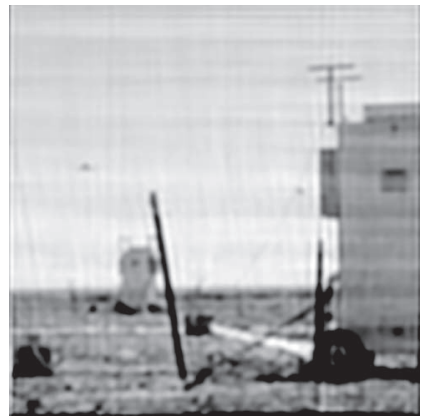

(h)
Fig. 5. (a) Example test image in [45], (b) Gaussian blurred image with $\sigma=6$ with PSNR $=24.05 \mathrm{~dB}$. (c) The image deblurred by the non-blind deconvolution method in [45]; PSNR $=22.43 \mathrm{~dB}$, (d) Result of the Ayers-Dainty algorithm; PSNR $=24.86 \mathrm{~dB}$, (e) Result of the Ayers-Dainty and phase information; PSNR $=24.91 \mathrm{~dB}$, (f) Ayers-Dainty and ESTV projection; PSNR $=24.74 \mathrm{~dB},(\mathrm{~g})$ Ayers-Dainty with phase and ESTV projections PSNR $=25.18 \mathrm{~dB}$. This is a sharp image but there are some ringing artifacts. (h) The restoration result of the non-blind approach based on phase only iterations; PSNR $=24.58 \mathrm{~dB}$. This is the only image clearly showing the white cable or stripe on the ground and the two antennas are clearly visible.

projection is shown in Fig. 5(f) with PSNR $=24.74 \mathrm{~dB}$. The restoration result of Ayers-Dainty method with phase and ESTV projections is shown in Fig. 5(g) with PSNR $=25.18 \mathrm{~dB}$. This is a sharp image but there are some ringing artifacts. In

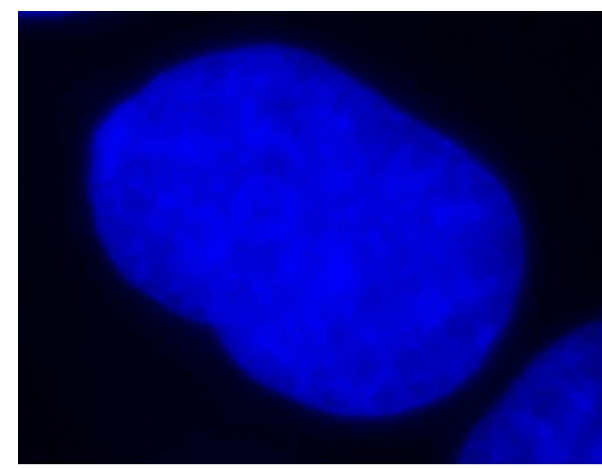

(a)

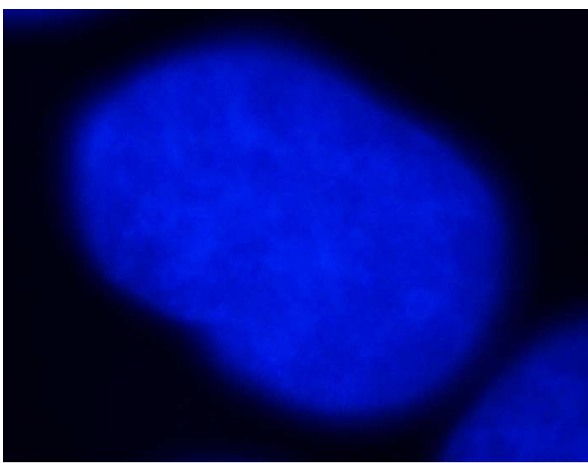

(b)

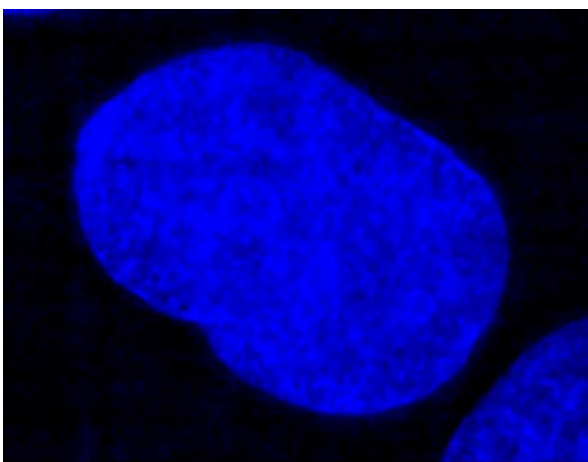

(c)

Fig. 6. (a) DAPI stained cell nuclei image (100X) from Huh7 cells with best possible focus: $x_{f}$ and (b) another image with slight out of focus $x_{g}$. The images $x_{f}$ and $x_{g}$ are combined to obtain (c) the deblurred image using Gaussian filtering based edge-enhancement together with phase and ESTV projections.

Fig. 5(h) the restoration result of a non-blind deconvolution method using the phase only iterations is shown. This has a low PSNR $=24.58 \mathrm{~dB}$ because the method did not produce a good result on the sky but it is the only image clearly showing the white cable or stripe on the ground and the two antennas are clearly visible.

\section{A. 3D Blind Deconvolution Examples}

In this subsection we present 3D examples. We combined two z-stack images to obtain a deconvolved image with clear features. The image shown in Fig. 6(c) is the result of a 3D edge enhancement algorithm together with FT phase and ESTV projections. The first image, $x_{f}$ shown in Fig. 6(a) is obtained by focusing the Nikon ECLIPSE Ti-S microscope. The image $x_{g}$ shown in Fig. 6(b) is obtained with a slight out-of focus. These are the images of Huh7, human hepatocellular carcinoma cells (ATCC), which were maintained in Dulbecco's Modified 


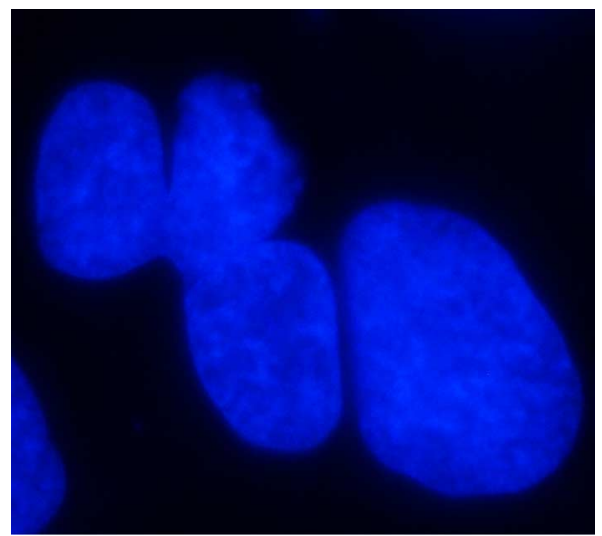

(a)

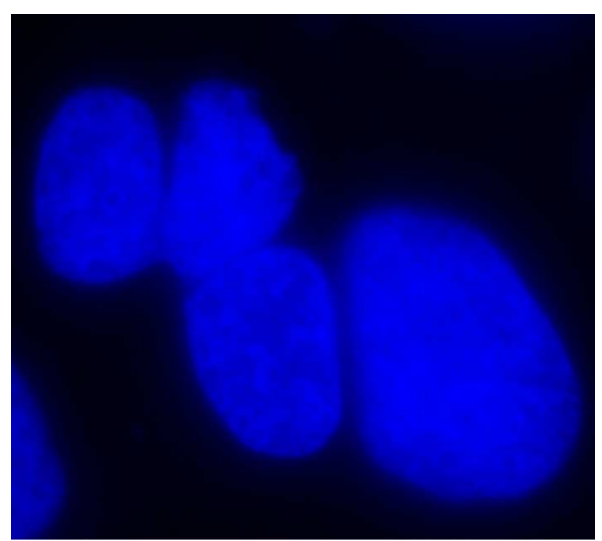

(b)

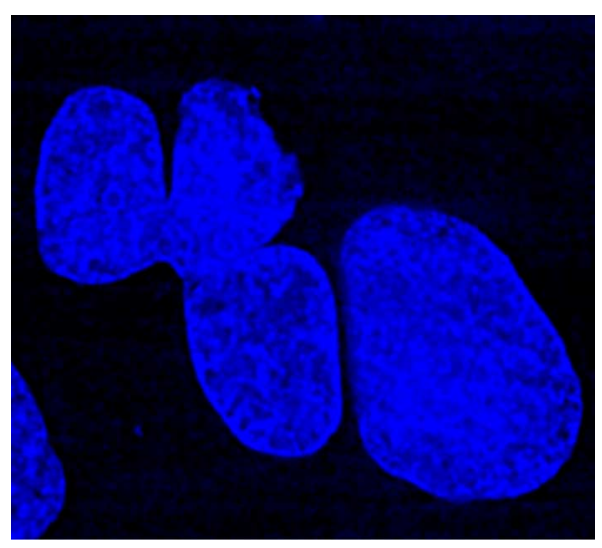

(c)

Fig. 7. DAPI stained cell nuclei image (100X) from Huh7 cells obtained with best possible focus: $x_{f}$ and (b) another image with slight out of focus $x_{g}$. The images $x_{f}$ and $x_{g}$ are combined to obtain (c) the deblurred image using Gaussian filtering based edge-enhancement together with phase and ESTV projections.

Eagle's Medium (DMEM) (Invitrogen GIBCO), supplemented with 10\% fetal bovine serum (FBS) (Invitrogen GIBCO), $2 \mathrm{mM}$ L-glutamine, $0.1 \mathrm{mM}$ nonessential amino acids, 100 units $/ \mathrm{mL}$ penicillin and $100 \mathrm{~g} / \mathrm{mL}$ streptomycin at $37^{\circ} \mathrm{C}$ in a humidified incubator under $5 \% \mathrm{CO}_{2}$.

Huh7 cells were stained using CD133 seeded onto coverslips (50000 cell/well) in 6-well plates and grown for 72 hours until cells reached $80 \%$ confluency. Cells were fixed with cold $4 \%$ paraformaldehyde for $30 \mathrm{~min}$ at room temperature and washed with 1 xPBS. For blocking cells were incubated with $3 \%$ BSA-PBS-T $(0.1 \%)$ for 90 minutes on a shaker at room

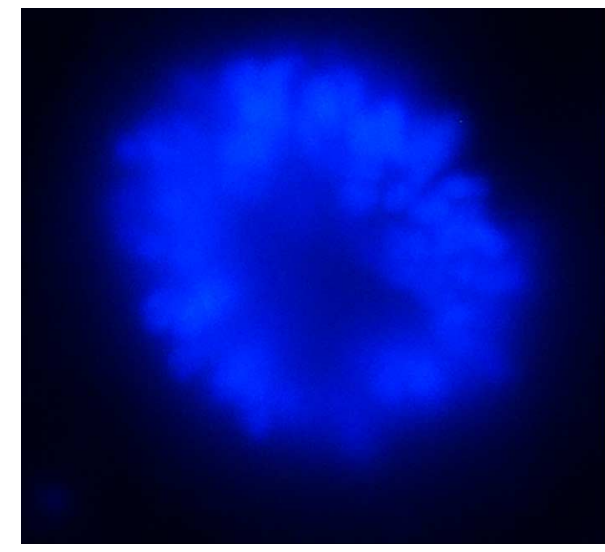

(a)

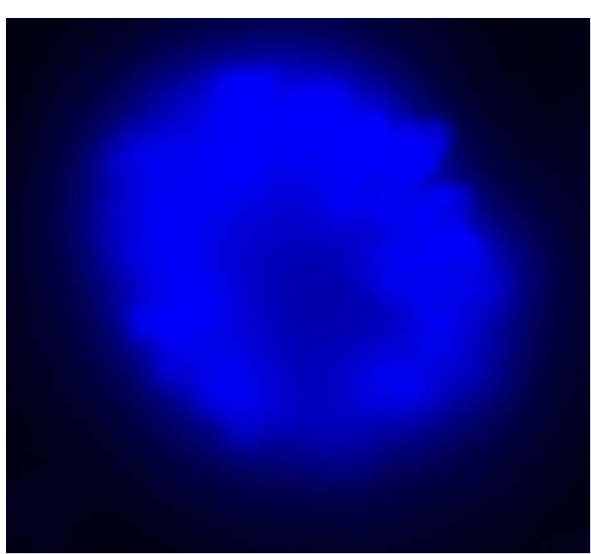

(b)

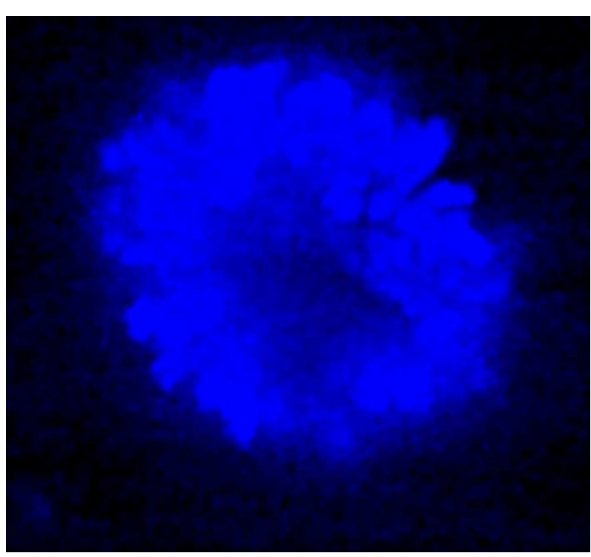

(c)

Fig. 8. The Huh7 cells nucleus image $(100 \times)$ obtained with best possible focus: $x_{f}$ and (b) another image with slight out of focus $x_{g}$. The images $x_{f}$ and $x_{g}$ are combined to obtain (c) the deblurred image using Gaussian filtering based edge-enhancement together with phase and ESTV projections.

temperature. Primary antibody incubation was done using human anti-CD133/2 (MACS cat.\# 130-090-851) for an hour as recommended by the manufacturer. Cells were washed 3 times with $1 \mathrm{xPBS}$ for 5 minutes. Secondary antibody incubation were done using Alexa-fluor 488 goat anti-mouse IgG antibody (Invitrogen cat.\# A11029, 1:1000) for an hour. After repeating the washing step, counterstaining (DAPI) was done using UltraCruz Mounting Medium (Santa Cruz cat.\# sc-24941).

Time-lapse images of fluorescently stained cells were taken using Nikon ECLIPSE Ti-S inverted microscopy and NIS-Elements Viewer software. Cell nuclei images shown 


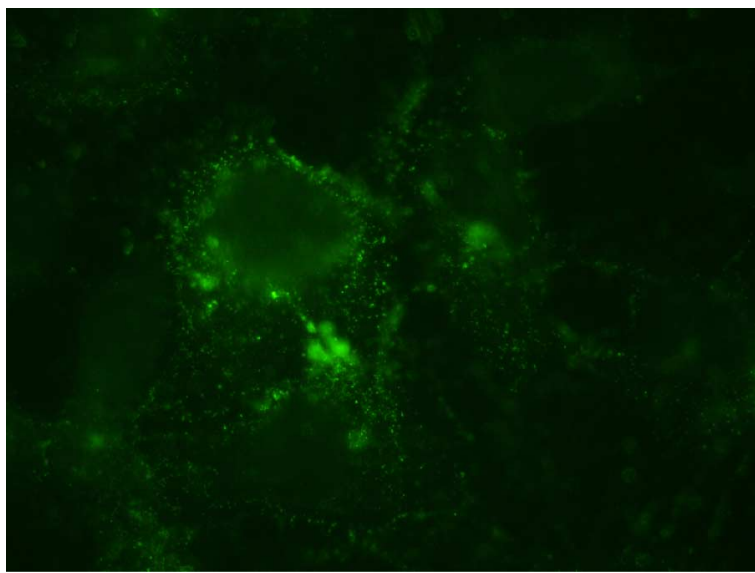

(a)

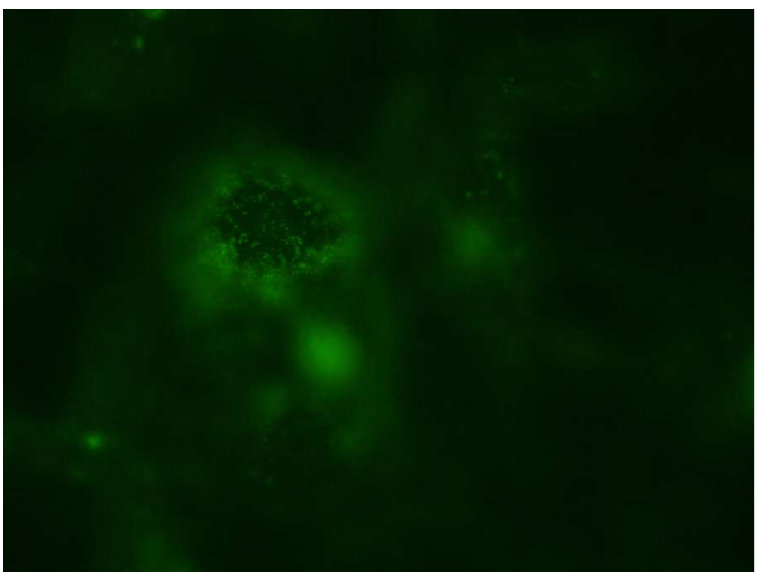

(b)

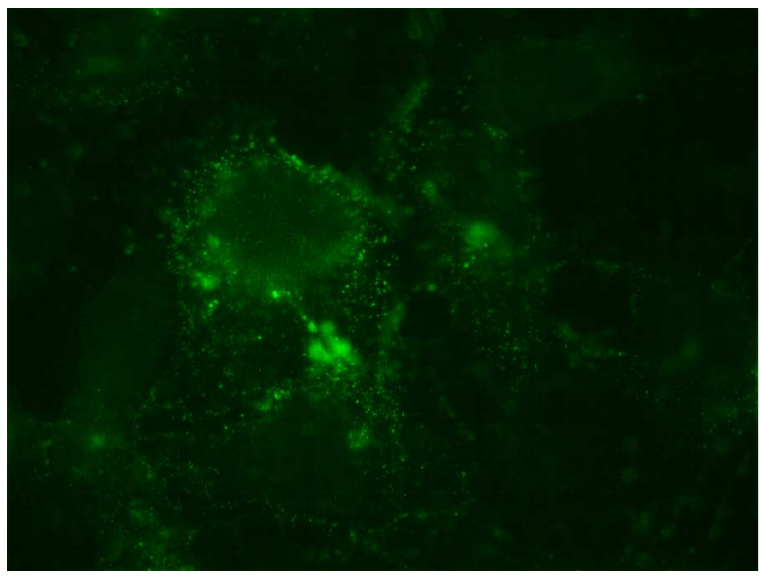

(c)

Fig. 9. Immunofluorescense images of CD133 positive Huh7 liver cancer cells with differential focus: (a) $x_{f}$ (top), (b) $x_{g}$ (middle). The images $x_{f}$ and $x_{g}$ are combined to obtain (c) the deblurred image using Gaussian filtering based edge-enhancement together with phase and ESTV projections.

in Fig. 6-8 were taken using 340-380 nm filter (duration: 1 minute, interval: $1 \mathrm{sec}$ ) under $150 \mathrm{~ms}$ exposure. CD133 positive cell time-lapse images shown in Fig. 9 were taken using the 465-495 nm filter (duration: 1 minute, interval: $1 \mathrm{sec}$ ) under $600 \mathrm{~ms}$ exposure.

The Gaussian filter based edge enhancement is achieved using the following equation:

$$
x_{e}=x_{f}+c_{h}\left(x_{g}-h * x_{g}\right)
$$

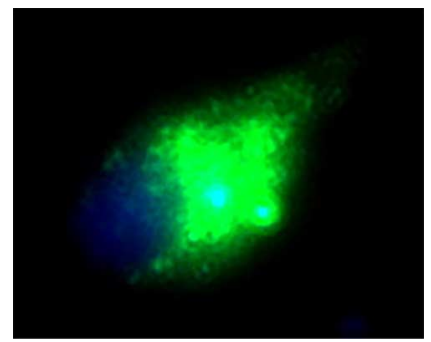

(a)

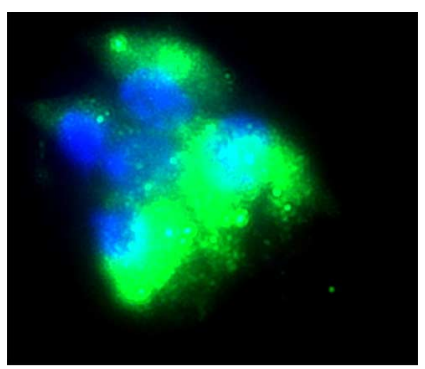

(c)

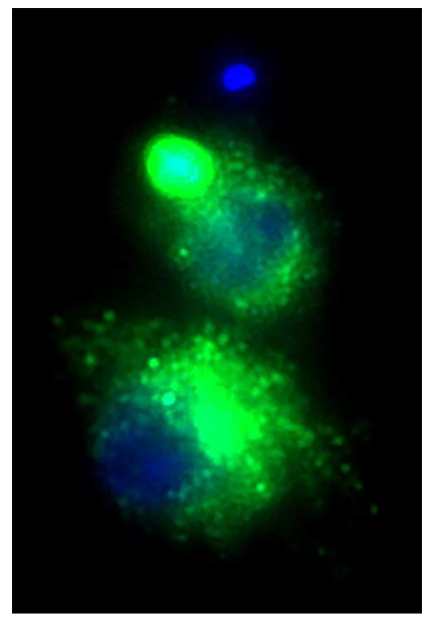

(b)

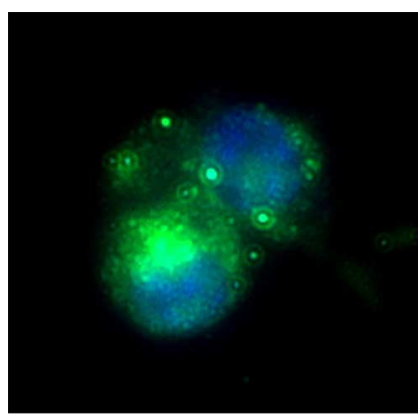

(d)
Fig. 10. Sample fluorescent labeled mouse liver tissue images used in experiments (a) Im-1, (b) Im-2 (c) Im-3, and (d) Im-4 obtained from [47].

where $h$ is a 2D Gaussian filter with $\sigma=5$ and $c_{h}=0.3$ is the high frequency component amplification coefficient. High frequency components of $x_{g}$ is added onto the better focused image $x_{f}$. After this step the edge-enhanced image $x_{e}$ is successively projected onto the phase and ESTV sets in an iterative manner. The image shown in Fig. 6(c) is obtained after 100 iterations.

Obviously, we cannot present PSNR values but the image shown in 6(c) has clearer features compared to 6(a). After 100 iterations we did not observe any improvements in 6(c).

In Fig. 7 another restoration example is shown. In Fig. 8 a third restoration example is shown. Clearly, axial information positively contributed to the deblurring process in Figs. 6, 7 and 8 .

In Fig. 9(a) and (b) two differentially focused immunofluorescence z-stack images of CD133 positive Huh7 liver cancer cells are shown, respectively. High frequency information from the image $x_{g}$ is combined with $x_{f}$ using (21) with $\sigma=10$ and $c_{h}=0.3$. The image shown in Fig. 9(c) is obtained using successive phase and ESTV projections after 20 set of iterations. As a result, blurred regions of $x_{f}$ are clearly visible in Fig. 9.

\section{B. 2D Simulated Blind Deconvolution Using Ayers-Dainty Method}

The contribution of phase and ESTV sets to a blind deconvolution problem is also evaluated using different fluorescence (FL) microscopy images obtained at Bilkent University as a part of a project funded by Turkish Scientific Research Council and German BMBF to track the motility and migration of cells. 
TABLE I

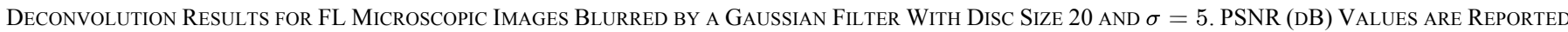

\begin{tabular}{|c|c|c|c|c|c|c|}
\hline Image & $\begin{array}{c}\text { Initial } \\
\text { PSNR }\end{array}$ & $\begin{array}{c}\text { Ayers- } \\
\text { Dainty }\end{array}$ & $\begin{array}{c}\text { Ayers-Dainty } \\
\text { with Phase }\end{array}$ & $\begin{array}{c}\text { Ayers Dainty } \\
\text { with ESTV }\end{array}$ & $\begin{array}{c}\text { Ayers Dainty } \\
\text { Phase\&ESTV }\end{array}$ & $\begin{array}{c}\text { Method } \\
\text { in [16] }\end{array}$ \\
\hline \hline im-1 & 32.08 & 34.25 & 34.51 & 34.56 & $\mathbf{3 5 . 1 9}$ & 31.43 \\
\hline im-2 & 31.86 & 31.59 & 31.81 & $\mathbf{3 2 . 6 7}$ & 32.13 & 31.17 \\
\hline im-3 & 32.62 & 33.24 & 33.10 & $\mathbf{3 4 . 3 1}$ & 33.57 & 30.59 \\
\hline im-4 & 33.75 & 31.78 & 31.58 & 30.92 & 29.78 & $\mathbf{3 3 . 8 5}$ \\
\hline im-5 & 35.66 & 37.51 & 35.86 & $\mathbf{3 7 . 8 2}$ & 36.08 & 34.10 \\
\hline im-6 & 33.37 & 36.21 & 35.89 & $\mathbf{3 6 . 4 6}$ & 36.15 & 32.59 \\
\hline im-7 & 35.03 & 38.76 & 38.53 & 39.70 & $\mathbf{4 0 . 2 4}$ & 32.32 \\
\hline im-8 & 34.64 & 38.33 & 37.72 & $\mathbf{3 9 . 2 6}$ & 38.27 & 32.71 \\
\hline im-9 & 31.14 & 31.86 & 31.55 & 32.12 & 32.08 & $\mathbf{3 2 . 7 1}$ \\
\hline im-10 & 33.48 & 36.81 & 36.84 & 37.56 & $\mathbf{3 8 . 2 2}$ & 32.16 \\
\hline im-11 & 35.17 & 35.50 & 38.57 & 35.43 & $\mathbf{3 9 . 6 5}$ & 34.63 \\
\hline im-12 & 34.64 & 30.68 & 34.87 & 32.65 & $\mathbf{3 6 . 7 4}$ & 34.82 \\
\hline im-13 & 35.35 & 36.52 & 38.00 & 37.05 & $\mathbf{3 9 . 1 0}$ & 32.26 \\
\hline im-14 & 36.44 & 36.41 & 37.84 & 36.98 & $\mathbf{3 8 . 5 7}$ & 33.32 \\
\hline
\end{tabular}

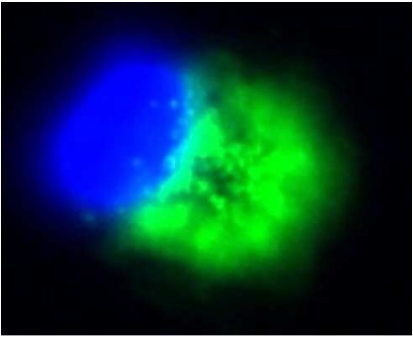

(a)

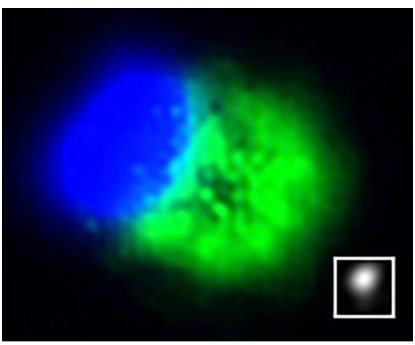

(c)

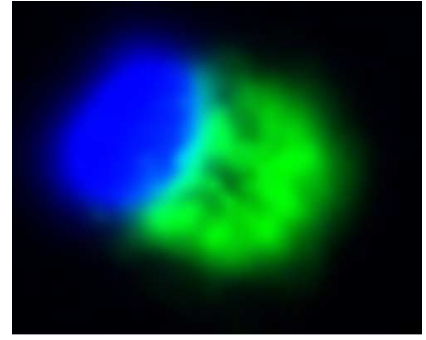

(b)

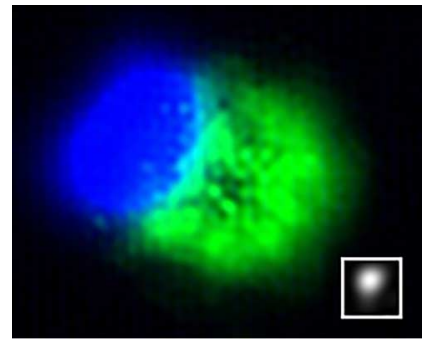

(d)

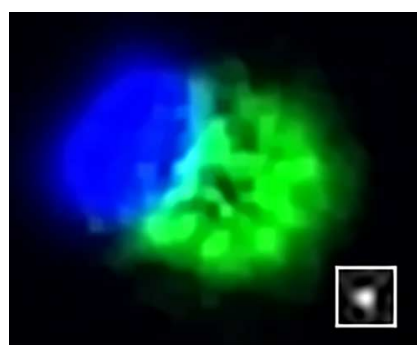

(e)

Fig. 11. Sample deblurring results for "im-7": (a) Original image, (b) blurred image (Gaussian $\sigma=5$ ); PSNR $=35.03 \mathrm{~dB}$, (c) Image obtained by Ayers-Dainty with Phase and ESTV sets; PSNR $=40.24 \mathrm{~dB}$, (d) Ayers-Dainty method; PSNR $=38.76 \mathrm{~dB}$, (e) image obtained using [16]; PSNR $=32.32 \mathrm{~dB}$. The blurring filter estimate for each case is shown in the bottom right corner.

The contact inhibition phenomena as a result of cell migration was first described in 1950s [46] in cultured cells which indicated that cell migration and motility are under the control of cell signaling. Cell migration and motility is a cellular activity that occurs during various stages of the life cycle of a cell under normal or pathological conditions. Embryonic development, wound-tissue healing, inflammation, angiogenesis, cancer metastasis are some of the major cellular activities that involve cell motility.

We used a video object tracker to track cells in our research. But the performance was very poor because the FL cell images were very smooth. Therefore we decided to develop a blind deconvolution method to obtain sharp cell images. After blind deconvolution, cells have clear features and sharper edges which can be used by video object trackers to track the motility of individual cells. In this application we do not have the z-stack images. We only have a slice of the FL image stack. The deconvolution operation is performed only the current image slice. The $2 \mathrm{D}$ image sequence is obtained using upright fluorescence microscope Nikon Eclipse 50i. We did not use its widefield mode but this microscope can be also used in widefield mode.

In order to evaluate PSNR we selected relatively sharp cell images from FL images and we synthetically blurred them using a $20 \times 20$ Gaussian filter with $\sigma=5$. We also visually checked the results of our algorithm on naturally blurred images. We tested proposed method against blind Ayers-Dainty [30] to observe the improvement. In Ayers-Dainty based methods, we started by an impulse image in which only one component was nonzero, as the initial guess. This way we ensured that all the frequency components would have a nonzero magnitude value. Furthermore we compared our method with another blind deconvolution method proposed in [16], which achieve deblurring by minimizing a regularization cost. Unfortunately, this method did not produce successful results in FL images.

In Fig. 10, some sample images are shown that are used in experimental studies. Ayers-Dainty method is compared with its own extension using FT phase and ESTV sets.

For Ayers-Dainty based methods, we limited the number of iterations to 300 and stopped the iterations when the estimation difference of consecutive iterations became smaller than a prescribed threshold. We have the results of standard Ayers-Dainty method [30], Ayers-Dainty and ESTV set, Ayers-Dainty and phase set, and the proposed Ayers-Dainty with phase and ESTV sets. The comparison of the PSNR performances of these algorithms is given in Table I.

We also used blind deconvolution method proposed in [16] to deblur FL microscopy images as shown in Table I. The PSNR 


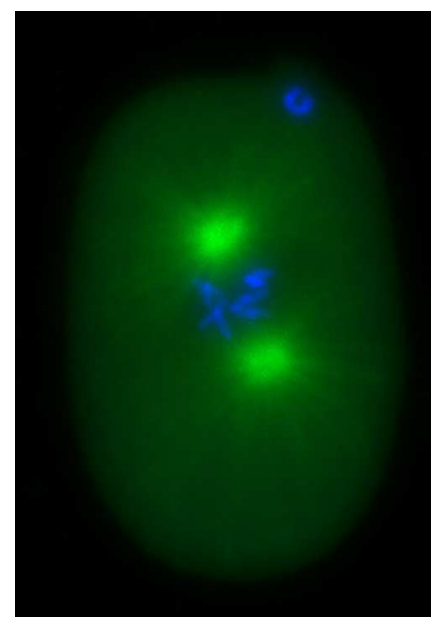

(a)

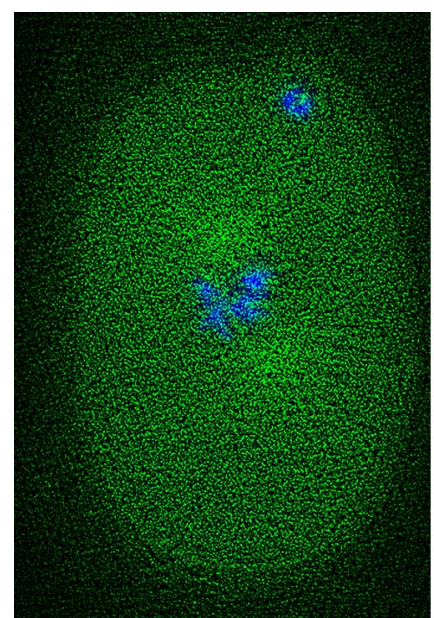

(c)

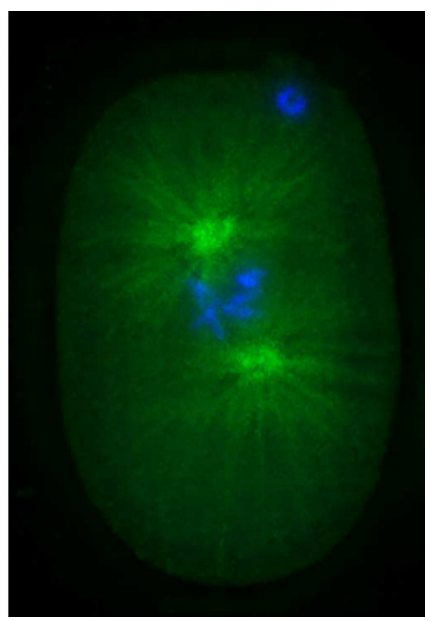

(b)

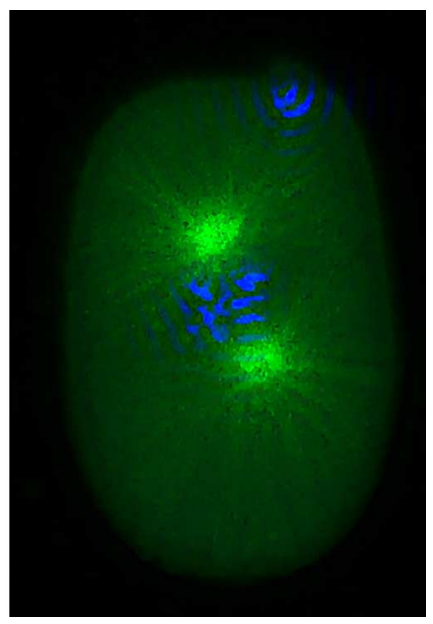

(d)
Fig. 12. The deconvolution results for FL image downloaded from [http://bigwww.epfl.ch/algorithms/mltldeconvolution/] (a) blurred image, (b) deblurred by the blind deconvolution using phase information (the images and the codes are provided in http://signal.ee.bilkent.edu.tr/BlindDeconvolution.html), (c) deblurred by Ayers and Dainty's algorithm, and (d) Deblurred by $\mathrm{Xu}$ et al.'s algorithm [7], which has clear artifacts in blue channels.

performance of this algorithm is not as good as the Ayers-Dainty method. Image deblurring results for "im-7" is shown in Fig. 11.

The bold PSNR values are the best results for a given image. We observed that best blind deconvolution results are obtained with Ayers-Dainty method using phase and ESTV set in general in our FL image test set. The method described in [16] cannot improve fine details of FL images as shown in Fig. 11 and Table I. In the following web-page you may find the MATLAB code of projections onto $\mathcal{C}_{\phi}$ and $\mathcal{C}_{\text {ESTV }}$ and example FL images which four of them are shown in Fig. 10. We have the Matlab source codes together with more examples in our web-page: http://signal.ee.bilkent.edu.tr/BlindDeconvolution.html.

In another set of experiments, we used the FL image shown in Fig. 12(a) which is blurred by an unknown filter or captured with a focus blur [48]. This image is deblurred using the blind deconvolution by phase information and its output is compared with Ayers and Dainty's and Xu et al.'s algorithm [7]. The deblurred image using the blind deconvolution by phase information and $\mathcal{C}_{\text {ESTV }}$, Ayers and Dainty's algorithm, and the $\mathrm{Xu}$ et al.'s algorithm are shown in Fig. 12(b), 12(c), and 12(d), respectively.
Ayers and Dainty's method sometimes does not converge as shown in Fig. 12(c). Xu et al.'s algorithm also diverges when we select "default" option. It does not diverge when we select "small" kernel option but the result is far from perfect as shown in Fig. 12. The blue channel has clear artifacts. Sets $\mathcal{C}_{\phi}$ and $\mathcal{C}_{\text {ESTV }}$ can be also incorporated into Xu et al.'s method for symmetric kernels but we do not have an access to the source code. We get the best results when we use $\mathcal{C}_{\phi}$ and $\mathcal{C}_{\text {ESTV }}$ in a successive manner as shown in Fig. 12(b).

\section{CONCLUSION}

Both FT phase and the epigraph set of the TV function are closed and convex sets. They can be used as a part of iterative microscopic image deblurring algorithms because blurring functions can be assumed to be symmetric in $x-y$ plane. Both sets not only provide additional information about the desired solution but they also stabilize the deconvolution algorithms. It is experimentally observed that phase and ESTV sets significantly improve the blind deblurring results of Ayers and Dainty's method in FL microscopy images. They can also be used as a part of non-blind deconvolution methods as well.

\section{ACKNOWLEDGMENT}

We would like to thank Editors of this Special Issue and the reviewers for their constructive comments.

\section{REFERENCES}

[1] P. Campisi and K. Egiazarian, Blind Image Deconvolution: Theory and Applications. Boca Raton, FL, USA: CRC, 2007.

[2] D. Kundur and D. Hatzinakos, "Blind image deconvolution," IEEE Signal Process. Mag., vol. 13, no. 3, pp. 43-64, May 1996.

[3] T. Chan and C.-K. Wong, "Total variation blind deconvolution," IEEE Trans. Image Process., vol. 7, no. 3, pp. 370-375, Mar. 1998.

[4] M. Sezan and H. Trussell, "Prototype image constraints for set-theoretic image restoration," IEEE Trans. Signal Process., vol. 39, no. 10, pp. 2275-2285, Oct. 1991.

[5] M. Sezan, "An overview of convex projections theory and its application to image recovery problems," Ultramicroscopy, vol. 40, no. 1, pp. 55-67, 1992.

[6] H. Trussell and M. Civanlar, "The feasible solution in signal restoration," IEEE Trans. Acoust., Speech, Signal Process., vol. 32, no. 2, pp. 201-212, Apr. 1984.

[7] L. Xu, S. Zheng, and J. Jia, "Unnatural 10 sparse representation for natural image deblurring," in Proc. IEEE Conf. Comput. Vis. Pattern Recog. (CVPR), Jun. 2013, pp. 1107-1114.

[8] P. Ye, H. Feng, Q. Li, Z. Xu, and Y. Chen, "Blind deconvolution using an improved 10 sparse representation," in Proc. 7th Int. Symp. Adv. Opt. Manuf. Testing Technol. (AOMATT'14), 2014, pp. 928 419-928 419, Int. Soc. for Opt. and Photon..

[9] J. Boulanger, C. Kervrann, and P. Bouthemy, "Adaptive spatio-temporal restoration for 4D fluorescence microscopic imaging," in Medical Image Computing and Computer-Assisted Intervention-MICCAI'05, ser. Lecture Notes in Computer Science, J. Duncan and G. Gerig, Eds. Berlin/Heidelberg, Germany: Springer , 2005, vol. 3749, pp. 893-901.

[10] C. Sorzano, E. Ortiz, M. López, and J. Rodrigo, "Improved Bayesian image denoising based on wavelets with applications to electron microscopy," Pattern Recog., vol. 39, no. 6, pp. 1205-1213, 2006.

[11] S. Acton, "Deconvolutional speckle reducing anisotropic diffusion," in Proc. IEEE Int. Conf. Image Process. (ICIP), Sep. 2005, vol. 1, pp. I-5-I-8.

[12] N. Dey, L. Blanc-Féraud, C. Zimmer, P. Roux, Z. Kam, J.-C. OlivoMarin, and J. Zerubia, "Richardson-Lucy algorithm with total variation regularization for 3D confocal microscope deconvolution," $M i$ croscopy Res. Tech., vol. 69, no. 4, pp. 260-266, 2006. 
[13] N. Dey, L. Blanc-Féraud, C. Zimmer, P. Roux, Z. Kam, J.-C. Olivo-Marin, and J. Zerubia, "3D microscopy deconvolution using Richardson-Lucy algorithm with total variation regularization," Res. Rep. RR-5272, 2004

[14] P. Pankajakshan, B. Zhang, L. Blanc-Féraud, Z. Kam, J.-C. OlivoMarin, and J. Zerubia, "Blind deconvolution for thin-layered confocal imaging," Appl. Optimiz., vol. 48, no. 22, pp. 4437-4448, Aug. 2009.

[15] B. Zhang, J. Zerubia, and J.-C. Olivo-Marin, "Gaussian approximations of fluorescence microscope point-spread function models," Appl. Optimiz., vol. 46, no. 10, pp. 1819-1829, Apr. 2007.

[16] D. Krishnan, T. Tay, and R. Fergus, "Blind deconvolution using a normalized sparsity measure," in Proc. IEEE Conf. Comput. Vis. Pattern Recog. (CVPR), 2011, pp. 233-240.

[17] E. Bostan, U. Kamilov, M. Nilchian, and M. Unser, "Sparse stochastic processes and discretization of linear inverse problems," IEEE Trans. Image Process., vol. 22, no. 7, pp. 2699-2710, Jul. 2013.

[18] P. L. Combettes and J.-C. Pesquet, "Image restoration subject to a total variation constraint," IEEE Trans. Image Process., vol. 13, no. 9, pp. 1213-1222, Sep. 2004.

[19] P. L. Combettes and J.-C. Pesquet, "Proximal splitting methods in signal processing," in Fixed-Point Algorithms for Inverse Problems in Science and Engineering, ser. Springer Optimization and Its Applications. New York, NY, USA: Springer, 2011, pp. 185-212.

[20] G. Chierchia, N. Pustelnik, J.-C. Pesquet, and B. Pesquet-Popescu, "Epigraphical projection and proximal tools for solving constrained convex optimization problems," Signal, Image, Video Process., pp. $1-13,2014$.

[21] A. E. Cetin and M. Tofighi, "Projection-based wavelet denoising [lecture notes]," IEEE Signal Process. Mag., vol. 32, no. 5, pp. 120-124, Sep. 2015.

[22] M. Tofighi, K. Kose, and A. E. Cetin, "Denoising images corrupted by impulsive noise using projections onto the epigraph set of the total variation function (PES-TV," Signal, Image, Video Process., vol. 9, no. 9, pp. 41-48, 2015.

[23] M. Tofighi, K. Kose, and A. E. Cetin, "Denoising using projections onto the epigraph set of convex cost functions," in Proc. IEEE Int Conf. Image Process. (ICIP), Oct. 2014, pp. 2709-2713.

[24] M. Hayes, J. Lim, and A. V. Oppenheim, "Signal reconstruction from phase or magnitude," IEEE Trans.Acoust., Speech, Signal Process., vol. ASSP-28, no. 6, pp. 672-680, Dec. 1980.

[25] A. V. Oppenheim and J. Lim, "The importance of phase in signals," Proc. IEEE, vol. 69, no. 5, pp. 529-541, May 1981.

[26] A. V. Oppenheim, M. H. Hayes, and J. S. Lim, "Iterative procedures for signal reconstruction from Fourier transform phase," Opt. Eng., vol. 21, no. 1, pp. $211122-211122,1982$.

[27] S. Curtis, J. Lim, and A. V. Oppenheim, "Signal reconstruction from one bit of Fourier transform phase," in Proc. IEEE Int. Conf. Acoust., Speech, Signal Process. (ICASSP)., Mar. 1984, vol. 9, pp. 487-490.

[28] L. I. Rudin, S. Osher, and E. Fatemi, "Nonlinear total variation based noise removal algorithms," Phys. D: Nonlinear Phenom., vol. 60, no. 1-4, pp. 259-268, 1992.

[29] A. Leclaire and L. Moisan, "No-reference image quality assessment and blind deblurring with sharpness metrics exploiting Fourier phase information," J. Math. Imag. Vis., vol. 52, no. 1, pp. 145-172, 2015.

[30] G. R. Ayers and J. C. Dainty, "Iterative blind deconvolution method and its applications," Opt. Lett., vol. 13, no. 7, pp. 547-549, Jul. 1988.

[31] D. Fish, J. Walker, A. Brinicombe, and E. Pike, "Blind deconvolution by means of the Richardson-Lucy algorithm," J. Opt. Soc. Amer. A, vol. 12, no. 1, pp. 58-65, 1995.

[32] N. Pustelnik, C. Chaux, and J. Pesquet, "Parallel proximal algorithm for image restoration using hybrid regularization," IEEE Trans. Image Process., vol. 20, no. 9, pp. 2450-2462, Sep. 2011.

[33] A. Chambolle, "An algorithm for total variation minimization and applications," J. Math. Imag. Vis., vol. 20, no. 1-2, pp. 89-97, Jan. 2004

[34] Y. Censor, W. Chen, P. L. Combettes, R. Davidi, and G. Herman, "On the effectiveness of projection methods for convex feasibility problems with linear inequality constraints," Comput. Optimiz. Applicat., vol. 51, no. 3, pp. 1065-1088, 2012.

[35] M. Tofighi, "Image restoration and reconstruction using projections onto epigraph set of convex cost functions," M.S. thesis, Bilkent Univ., Ankara, Turkey, 2015.

[36] A. E. Cetin, A. Bozkurt, O. Gunay, Y. H. Habiboglu, K. Kose, I. Onaran, R. A. Sevimli, and M. Tofighi, "Projections onto convex sets (POCS) based optimization by lifting," in Proc. IEEE Global Conf. Signal Inf. Process. (GlobalSIP), 2013, p. 623.
[37] D. Youla and H. Webb, "Image restoration by the method of convex projections: Part 1 theory," IEEE Trans. Med. Imag., vol. MI-1, no. 2, pp. 81-94, 1982.

[38] A. E. Cetin and A. Tekalp, "Robust reduced update Kalman filtering," IEEE Trans. Circuits Syst., vol. 37, no. 1, pp. 155-156, Jan. 1990.

[39] A. E. Çetin and R. Ansari, "Convolution-based framework for signal recovery and applications," J. Opt. Soci. Amer., vol. 5, no. 8, pp. 1193-1200, Aug. 1988

[40] H. Trussell and M. R. Civanlar, "The Landweber iteration and projection onto convex set," IEEE Trans. Acoust., Speech, Signal Process., vol. ASSP-33, no. 6, pp. 1632-1634, 1985.

[41] H. Stark, D. Cahana, and H. Webb, "Restoration of arbitrary finiteenergy optical objects from limited spatial and spectral information," J. Opt. Soc. Amer., vol. 71, no. 6, pp. 635-642, Jun. 1981.

[42] P. Combettes, "The foundations of set theoretic estimation," Proc. IEEE, vol. 81, no. 2, pp. 182-208, Feb. 1993.

[43] S. Theodoridis, K. Slavakis, and I. Yamada, "Adaptive learning in a world of projections," IEEE Signal Process. Mag., vol. 28, no. 1, pp. 97-123, Jan. 2011.

[44] I. Yamada, "The hybrid steepest descent method for the variational inequality problem over the intersection of fixed point sets of nonexpansive mappings," in Inherently Parallel Algorithms in Feasibility and Optimization and their Applications, ser. Studies in Computational Mathematics. Amsterdam, The Netherlands: Elsevier, 2001, vol. 8, pp. 473-504.

[45] B. Dougherty, 1999, MS Windows NT Iterative Deconvolution [Online]. Available: http://www.optinav.com/Iterative-Deconvolution.htm

[46] M. Abercrombie and J. E. Heaysman, Invasiveness of Sarcoma Cells. New York, NY, USA: Nature Publishing Group, 1954.

[47] E. Akhan, D. Tuncel, and K. C. Akcali, "Nanoparticle labeling of bone marrow-derived rat mesenchymal stem cells: Their use in differentiation and tracking," BioMed Res. Int., vol. 2015, p. 9, 2015.

[48] C. Vonesch and M. Unser, "A fast multilevel algorithm for waveletregularized image restoration," IEEE Trans. Image Process., vol. 18 no. 3, pp. 509-523, Mar. 2009.

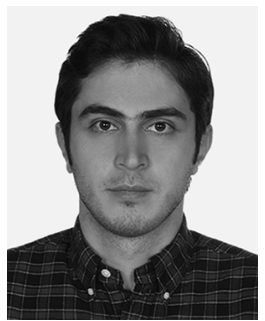

Mohammad Tofighi received his B.Sc. in electrical engineering from Urmia University, Urmia, Iran, in 2012. In 2010, he collaborated with the Advanced Communication Research Institute, Department of Electrical Engineering, Sharif University of Technology, Tehran, Iran. He received his M.Sc. in electrical engineering from the Electrical and Electronics Engineering Department, Bilkent University, Ankara, Turkey, in 2015. He is currently pursuing his $\mathrm{PhD}$ in Department of Electrical Engineering, Pennsylvania State University, University Park, PA. His research interests include signal and image processing, computer vision and machine learning.

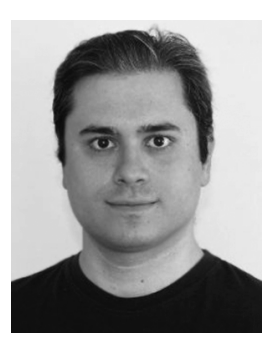

Onur Yorulmaz received his B.Sc. and M.Sc. degree in the Electrical and Electronics Engineering Department, Bilkent University, Ankara, Turkey, in 2012 $\mathrm{He}$ is currently working on his $\mathrm{PhD}$ studies in the Electrical and Electronics Engineering Department of Bilkent University. His research interests include signal and image processing, computer vision, and machine learning.

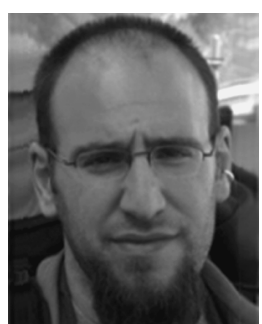

Kivanç Köse got his Ph.D. degree from the Electrical and Electronics Engineering Department, Bilken University in 2011. He is currently working as a Postdoctoral Research Fellow at Dermatology Department, Memorial Sloan-Kettering Cancer Center. $\mathrm{He}$ is an associate editor of Signal, Image and Video Processing journal. He research interests include signal and image processing, inverse problems in image processing, computer vision and machine learning in medical image processing applications. 


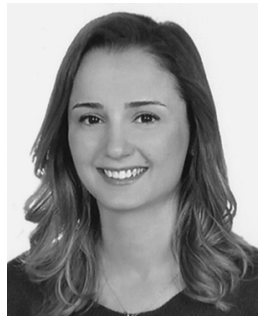

Deniz Cansen Yıldırım received her B.Sc. in molecular biology and genetics from Bilkent University Ankara, Turkey, in 2011. She is currently carrying out her Ph.D. studies at the same department in Bilkent University. Her research interests include discovery of novel small molecule kinase inhibitors against liver cancer and cancer stem cells.

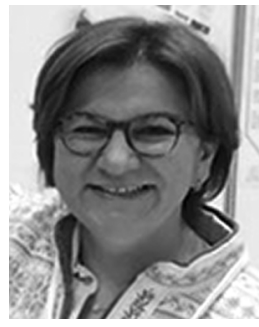

Rengül Cetin-Atalay received her Ph.D. degree in 1997 from Université de Paris-Sud, Orsay, France. She was a French Government scholar during her $\mathrm{PhD}$. She carried out her doctoral studies as a Research Assistant at Ecole Polytechnique, Paris, France. Between 1997-2014, Dr. Cetin-Atalay was a faculty member at Bilkent University. She worked as an Assistant Professor at the Virginia Bioinformatics Institute, Virginia Polytechnic Institute and State University, Virginia, USA, during her sabbatical leave in 2004. She is currently a faculty member at
Middle East Technical University, Ankara, Turkey. Dr. Cetin-Atalay's research interests lie in the fields of bioinformatics and molecular cellular biology of liver cancer.

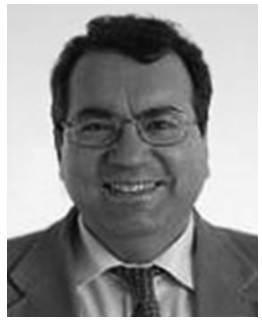

A. Enis Cetin got his Ph.D. degree from the University of Pennsylvania in 1987. Between 1987-1989, he was Assistant Professor of Electrical Engineering at the University of Toronto. He has been with Bilkent University, Ankara, Turkey, since 1989. $\mathrm{He}$ will be on sabbatical leave at University of California, San Diego, starting Dec. 2015. He was an Associate Editor of the IEEE TRANSACTIONS ON IMAGE PROCESSING between 1999-2003. Currently, he is the editor in-chief of Signal, Image and Video Processing journal and on the editorial boards of journals Signal Processing and Journal of Advances in Signal Processing (EURASIP), and Journal of Machine Vision and Applications (IAPR), Springer. $\mathrm{He}$ is currently an Associate Editor of the IEEE Signal Processing Magazine and IEEE TRANSACtions on Circuits, Systems, and Video TeChNology. $\mathrm{He}$ is a fellow of IEEE. His research interests include signal and image processing, human-computer interaction using vision and speech, audio-visual multimedia databases. 